\title{
On Aggregative and Mean Field Games with Applications to Electricity Markets
}

\author{
Dario Paccagnan, Maryam Kamgarpour and John Lygeros ${ }^{1}$
}

\begin{abstract}
We study the existence and uniqueness of Nash equilibria for a certain class of aggregative games with finite and possibly large number of players. Sufficient conditions for these are obtained using the theory of variational inequalities together with the specific structure of the objective functions. We further present an algorithm that converges to the Nash equilibrium in a decentralized fashion with provable guarantees. The theoretical results are applied to the problem of managing the charging of a large fleet of plug-in electric vehicles and the results are compared with the existing work.
\end{abstract}

\section{INTRODUCTION}

Technological advances in recent years have paved the way for application of control to increasingly more complex and large scale dynamical systems. Examples include increased autonomy in ground and air transportation systems, multiagent vehicle networks, and the power grid system [1]. A main challenge in developing control systems of the future is to consider individual objectives of each agent while taking into account local and global constraints and costs. Furthermore, control algorithms need to be decentralized in the sense that each agent should take its decision using local information and limited partial information on the state of the global system. Motivated by these problems, the purpose of this paper is to analyze and design a decentralized algorithm for a specific class of aggregative games.

Game theory is a powerful framework to capture interaction of a large number of self optimizing agents with coupled objectives or constraints, but it becomes intractable as the number of players increases. Motivated by this shortcoming, mean field game theory studies a simplified model obtained by letting the number of players tend to infinity [2], [3] Based on this approach, algorithms are derived which are decentralized in the sense that each agent uses its local information and the mean of the state of other agents [3]. Such algorithms ensure that the solution for finite number of players is $\varepsilon$-Nash optimal, where $\varepsilon$ approaches zero as the number of players approach infinity. The liberalized electricity market of the future can be modelled as a game between a large number of profit maximizing generators or consumers. Thus, mean field game theory is increasingly applied in this domain [4], [5], [6].

One of the challenges for the electricity market and for the grid operation is how to handle the introduction of plugin electric vehicles (PEVs) on a large scale. The problem

This work was supported by Swiss Nano-Tera project HeatReserves and Swiss Competence Centers for Energy Research FEEB\&D.

${ }^{1}$ D. Paccagnan, M. Kamgarpour and J. Lygeros are with the Automatic Control Laboratory, ETH Zürich, Switzerland. Email: \{dariop, mkamgar, lygeros\}econtrol.ee.ethz.ch of coordinating the charging of PEVs was discussed initially in [4], [7], [8]. Here, the authors proved that the centralized coordination of PEVs results in a solution which exhibits charging during low-demand hours and thus, can alleviate demand peaks. Furthermore, [4], [7] illustrated that this solution can be obtained as the Nash equilibrium (NE) of a game with an infinite number of homogenous players. In the finite population size such algorithm results in $\varepsilon$-Nash performance. The authors in [9] expanded this idea to show that under a more relaxed set of assumptions, $\varepsilon$-Nash equilibria can still be obtained. An unaddressed problem is whether such games possess a NE for finite population size and if so, to design a decentralized algorithm to converge to it.

In this paper, we present a new approach to address the problems above. In particular, inspired from the recent advances in variational inequalities [10], we show that the aggregative games studied in [4], [9] do possess a unique Nash equilibrium even in finite population size. Furthermore we design a decentralized algorithm that converges to this NE. This decentralized algorithm has the same information structure as in the above mentioned works, in that a central coordinator broadcasts the mean to the population but results in Nash performance instead of $\varepsilon$-Nash optimality. Our point of view is that for deterministic aggregative games, in contrast to stochastic ones [2], [3] one can obtain desired analysis without restoration to the mean field limit. We apply our framework to the PEV charging problem and compare the results with previous works along this line.

Our contributions are threefold. First, we study the quadratic game of [9] to show existence and uniqueness of a NE under suitable conditions. Second, we generalize the results to a classes of nonlinear aggregative games. Third, we provide a decentralized algorithm that converges to the NE. In terms of application, we consider the PEV charging problem and show how our approach eliminates the need of an artificial quadratic term in the individual cost functions, which was needed in the convergence proofs of [4], [9].

The paper is organized as follows. In Section II we describe the problem and use tools from variational inequalities to study the NE. In Section III, we present decentralized algorithms to converge to the NE. In section IV we illustrate the application of our framework to the charging problem. In Section V we conclude and summarize future work.

\section{Notation}

$\mathbb{N}, \mathbb{R}$ denote respectively the set of natural and real numbers; $\mathcal{H}$ represents the Hilbert space with inner product $\langle\cdot, \cdot\rangle: \mathcal{H} \times \mathcal{H} \rightarrow \mathbb{R}$ and norm $\|\cdot\|_{2}:=\sqrt{\langle\cdot, \cdot\rangle} ; \mathrm{Id}: \mathcal{H} \rightarrow \mathcal{H}$ 
represents the identity operator. DT: $\mathbb{R}^{n} \rightarrow \mathbb{R}^{n \times n}$ indicates the Jacobian of the differentiable map $T: \mathbb{R}^{n} \rightarrow \mathbb{R}^{n} ; \nabla_{x} g$ : $\mathbb{R}^{n} \rightarrow \mathbb{R}^{n}$ indicates the gradient of the differentiable map $g: \mathbb{R}^{n} \rightarrow \mathbb{R} ;\left(\nabla_{x_{i}} J_{i}\right)_{i=1}^{N}: \mathbb{R}^{n} \rightarrow \mathbb{R}^{n N}$ denotes the stacked vector of the gradients $\nabla_{x_{i}} J_{i}$ for differentiable $J_{i}: \mathbb{R}^{n} \rightarrow$ $\mathbb{R}, i=1, \ldots, N . I_{n}, \mathbb{1}_{n}$, denote the $n$-dimensional identity matrix and vector of unit entries, respectively. The operation $A \otimes B$ denotes the Kronecker product of matrices $A, B$. Given $A \in \mathbb{R}^{n \times n}$ not necessarily symmetric, we denote $A \succ$ $0(\succeq 0) \Longleftrightarrow x^{\top} A x>0(\geq 0), \forall x \neq 0$. Spec $[A]$ represents the multiset of eigenvalues of the matrix $A$ counted with multiplicity, while $\lambda_{\max }(A), \lambda_{i}(A)$ denote respectively the maximum and the $i$-th eigenvalue of the symmetric matrix $A$ arranged in descending order. The notation $\varepsilon \sim \mathcal{O}\left(N^{\alpha}\right)$ with $\alpha \in \mathbb{R}$ indicates that there exists finite $l>0$ such that $\lim _{N \rightarrow \infty} \varepsilon N^{-\alpha}=l$. Given a vector $\left[x_{1}, \ldots, x_{N}\right] \in \mathbb{R}^{m N}$, $x_{-i}:=\left[x_{1}, \ldots, x_{i-1}, x_{i+1}, \ldots, x_{N}\right] \in \mathbb{R}^{m(N-1)}$; given a set $K=\prod_{j=1}^{N} K_{j}, K_{-i}:=\prod_{j \neq i}^{N} K_{j}$. The uniform distribution on the real interval $[a, b]$ is denoted by $\mathrm{U}([a, b])$.

\section{AGGREGATIVE GAMES AND VARIATIONAL INEQUALITIES}

In this section we study a certain class of aggregative games and address existence and uniqueness of a NE. We first present some tools from the theory of variational inequalities and successively apply them to the specific game.

\section{A. Game formulation}

Let us consider a single shot game with $N$ players, where each of them aims at minimizing a cost function that depends on its private decision and on the average decision of the others. In particular, consider a non-cooperative game where the payoff of each player $J_{i}\left(x_{i}, x_{-i}\right): \mathbb{R}^{N m} \rightarrow \mathbb{R}$ is

$$
J_{i}\left(x_{i}, x_{-i}\right)=x_{i}^{\top} Q x_{i}+g\left(\frac{1}{N} \sum_{j=1}^{N} x_{j}\right)^{\top} x_{i},
$$

where $g: \mathbb{R}^{m} \rightarrow \mathbb{R}^{m}$ is twice differentiable. The decision variable of the $i$-th player is $x_{i} \in K_{i}$, where $K_{i}$ is a closed and convex subset of $\mathbb{R}^{m}$. In the above, $Q \in \mathbb{R}^{m \times m}, i=$ $1, \ldots, N$. We refer to this aggregative game as $\mathcal{G}_{A}$ [11]. Note that $\mathcal{G}_{A}$ includes a large class of deterministic finite horizon dynamic games for which one is interested in open loop Nash equilibria, such as linear quadratic games [12]. For the PEV charging problem, this reformulation is detailed in section IV. The function $g$ can be interpreted as the marginal price of electricity, which in a liberalized market is modelled through dependence on aggregated production and consumption [4], [6]. When $Q=0, J_{i}$ can be thought of as the price of buying $x_{i}$ units of electricity and the number of players $N$ can be large due to participation of multiple consumers/producers.

\section{B. Tools from Variational Inequalities}

We will introduce the connection between variational inequalities and Nash equilibria in non-cooperative games. In Section II-C and II-D we will apply this tool to game $\mathcal{G}_{A}$.

Definition 1 (Variational Inequality): Given a subset $K \subset \mathcal{H}$ and an operator $T(\cdot): \mathcal{H} \rightarrow \mathcal{H}$, the Variational
Inequality (VI) problem denoted with $V I(K, T)$ is to find $x^{\star} \in K$ such that $\left\langle T\left(x^{\star}\right), x-x^{\star}\right\rangle \geq 0 \quad \forall x \in K$.

This problem is connected with several areas of mathematics such as optimization, game theory and fixed point theory. For a discussion when $\mathcal{H}$ is finite dimensional see for example [10]. Depending on the properties of $T(\cdot)$, one can prove existence and uniqueness of the solution of $V I(K, T)$ as per Theorem 1, see [13]. A key role is played by monotonicity.

Definition 2 (Monotone Operator): The operator $T(\cdot)$ : $\mathcal{H} \rightarrow \mathcal{H}$ is said to be monotone (resp. strictly monotone) if $\langle T(x)-T(y), x-y\rangle \geq 0$ (resp. if $\langle T(x)-T(y), x-y\rangle>0$ ) $\forall x \neq y \in \mathcal{H}$.

From now on, $\langle\cdot, \cdot\rangle$ represents the usual inner product on $\mathbb{R}^{n}$.

Theorem 1 (Existence and Uniqueness for VIs): Let $T(\cdot)$ be a monotone, continuous operator from $\mathbb{R}^{n}$ into itself and let $K$ be a bounded closed convex subset of $\mathbb{R}^{n}$. Then, the set of all solutions of $\operatorname{VI}(K, T)$ is a nonempty closed convex subset of $K$. If furthermore $T(\cdot)$ is strictly monotone, then the solution to $\operatorname{VI}(K, T)$ is unique.

It can be verified that a differentiable operator $T(\cdot): \mathbb{R}^{n} \rightarrow$ $\mathbb{R}^{n}$ is (strictly) monotone if the Jacobian (DT $(x) \succ 0$ ) $\mathrm{D} T(x) \succeq 0 \forall x \in \mathbb{R}^{n},[14]$.

We now present the connection between NE and the theory of variational inequalities [15].

Definition 3 (Nash Equilibrium): A point $x^{\star} \in K$ is called a Nash equilibrium of the game $\mathcal{G}_{A}$ if

$$
J_{i}\left(x_{i}^{\star}, x_{-i}^{\star}\right) \leq J_{i}\left(x_{i}, x_{-i}^{\star}\right) \quad \forall x_{i} \in K_{i} \quad \forall i=1, \ldots, N .
$$

Theorem 2 (Nash equilibria and VIs): Suppose for the above game $\mathcal{G}_{A}$ the non empty strategy sets $K_{i}$ are closed and convex and that for every fixed $x_{-i} \in K_{-i}$ the payoff functions $J_{i}\left(x_{i}, x_{-i}\right)$ are convex and continuously differentiable in $x_{i} \in K_{i}$. Then a point $x^{\star} \in K$ is a Nash equilibrium for $\mathcal{G}_{A}$ if and only if it is a solution of the variational inequality $V I(K, T)$, with $K=\prod_{i} K_{i}$ and

$$
T(x)=\left(\nabla_{x_{i}} J_{i}\left(x_{i}, x_{-i}\right)\right)_{i=1}^{N} .
$$

\section{Existence and uniqueness of $N E$ : the affine case}

Let us consider the game $\mathcal{G}_{A}$. First, we restrict our attention to $g: \mathbb{R}^{m} \rightarrow \mathbb{R}^{m}$ affine. Equation (1) becomes

$$
J_{i}\left(x_{i}, x_{-i}\right)=x_{i}^{\top} Q x_{i}+2\left(C \frac{1}{N} \sum_{j=1}^{N} x_{j}+c\right)^{\top} x_{i},
$$

with $C \in \mathbb{R}^{m \times m}, c \in \mathbb{R}^{m}$. This is the formulation in [9]. Using the theory of VIs, we prove existence and uniqueness of the Nash equilibrium for the aggregative game $\mathcal{G}_{A}$ with $J_{i}\left(x_{i}, x_{-i}\right)$ as in (3). Given the structure of (3), the operator $T(x)$ defined in (2) takes the form

$$
\begin{gathered}
T(x)=b+\left(A_{1}+A_{2}\right) x \quad \text { where } \quad b=\mathbb{1}_{N} \otimes 2 c, \\
A_{1}=I_{N} \otimes\left(Q+Q^{\top}+\frac{2 C^{\top}}{N}\right), \quad A_{2}=\left(\mathbb{1}_{N} \mathbb{1}_{N}^{\top}\right) \otimes \frac{2 C}{N} .
\end{gathered}
$$

Proposition 1: If $Q \succ 0$ and $C \succeq 0$ or if $Q \succeq 0$ and $C \succ 0$, the set of Nash equilibria of $\mathcal{G}_{A}$ coincides with the 
set of solutions of $V I(K, T)$ with $K=\prod_{i=1}^{N} K_{i}$ and $T(x)$ as in equation (4). Furthermore, $T(x)$ is strictly monotone and $\mathcal{G}_{A}$ has a unique NE.

Proof: By hypothesis each $K_{i}$ is closed and convex, while for fixed $\hat{x}_{-i} \in K_{-i}$ the $i$-th player payoff reduces to $J_{i}\left(x_{i}\right)=x_{i}^{\top}\left(Q+\frac{2 C^{\top}}{N}\right) x_{i}+2\left(C \frac{1}{N} \sum_{j \neq i}^{N} \hat{x}_{j}+c\right)^{\top} x_{i}$ which is continuously differentiable and convex in $x_{i} \in K_{i}$ since $Q+$ $\frac{2 C^{\top}}{N} \succ 0$. Indeed, using the definition of a convex function, one can show for a generic non symmetric matrix $A$ that $A \succeq$ $0 \Longrightarrow y(x)=x^{\top} A x+b^{\top} x$ convex. Here, $A=Q+\frac{2 C}{N}^{\top} \succ$ 0 because $x^{\top}\left(Q+\frac{2 C}{N}^{\top}\right) x=x^{\top} Q x+\frac{2}{N} x^{\top} C x>0$ when $x \neq 0$, by the hypothesis on $Q, C$. Thus, by Theorem 2 the game $\mathcal{G}_{A}$ is equivalent to solving the $V I(K, T)$ with $T(x)=$ $\left(\nabla_{x_{i}} J_{i}\left(x_{i}, x_{-i}\right)\right)_{i=1}^{N}$. Given the structure of $J_{i}\left(x_{i}, x_{-i}\right)$, one can compute explicitly $T(x)$ as in (4). The operator $T(\cdot)$ is strictly monotone. Indeed its Jacobian is positive definite, since $\mathrm{D} T(x)=A_{1}+A_{2}$, and $x^{\top}\left(A_{1}+A_{2}\right) x=\sum_{i=1}^{N} x_{i}(Q+$ $\left.Q^{\top}+\frac{2 C^{\top}}{N}\right) x_{i}^{\top}+\left(\sum_{i=1}^{n} x_{i}\right)^{\top} \frac{2 C}{N}\left(\sum_{i=1}^{n} x_{i}\right)>0$ when $x \neq 0$ if $Q \succ 0$ and $C \succeq 0$ or $Q \succeq 0$ and $C \succ 0$. By Theorem 1 we conclude that the solution to $V I(K, T)$ is unique and so is the Nash equilibrium for $\mathcal{G}_{A}$.

\section{Existence and uniqueness of NE: the nonlinear case}

Proposition 2: Assume that the function $g(\cdot): \mathbb{R}^{m} \rightarrow \mathbb{R}^{m}$ is strictly monotone, that each component $g_{r}(\cdot)$ is convex in the argument, $x_{i} \geq 0$ and $Q \succeq 0$. Then, the NE of $\mathcal{G}_{A}$ are the solutions to $V I(K, T)$, with $K=\prod_{i} K_{i}$, $T(x)=\left(\nabla_{x_{i}} J_{i}\left(x_{i}, x_{-i}\right)\right)_{i=1}^{N}$. $\quad$ Proof: $\quad$ Each strategy set $K_{i}$ is non empty, convex and closed. Furthermore, the payoff functions $J_{i}\left(x_{i}, x_{-i}\right)$ are continuously differentiable. The Hessian of $J_{i}\left(x_{i}, x_{-i}\right)$ with respect to $x_{i}$ is given by

$$
\begin{gathered}
H_{i}\left(x_{i}, x_{-i}\right)=Q+Q^{\top}+\frac{1}{N} \mathrm{D} g(\sigma(x))^{\top}+ \\
+\frac{1}{N} \mathrm{D} g(\sigma(x))+\frac{1}{N^{2}} \sum_{r=1}^{m} H^{r}(\sigma(x)) x_{i}^{r},
\end{gathered}
$$

where $\sigma(x)=\frac{1}{N} \sum_{j=1}^{N} x_{j}, \mathrm{D} g(\sigma(x)) \in \mathbb{R}^{m \times m}$ and $H^{r}(\sigma(x)) \in \mathbb{R}^{m \times m}$ are respectively the Jacobian of $g$ and the Hessian of $g_{r}(\sigma)$ with respect to $\sigma$. Strict monotonicity of $g(\cdot)$ implies $\mathrm{D} g(\sigma(x)) \succ 0$, while $g_{r}(\cdot)$ convex implies $H^{r}(\sigma(x)) \succ 0$. Since $Q \succeq 0$ by hypothesis, the Hessian in (5) is positive definite and consequently $J_{i}\left(x_{i}, x_{-i}\right)$ is convex in $x_{i} \in K_{i}$. Theorem 1 gives the desired result. Uniqueness is guaranteed by Theorem 1 if $\mathrm{D} T(x) \succ 0$.

Remark 1: The differences between this approach and those in [4], [9] are as follows. In these previous works the authors prove the existence of an $\varepsilon$-Nash with $\varepsilon \sim$ $\mathcal{O}(1 / N)$. Here we prove existence and uniqueness for the Nash equilibrium for all finite $N$. Furthermore, it is no longer necessary to have an additional quadratic term $Q \succ 0$ as in the works mentioned above to guarantee existence and uniqueness. When $Q=0$ Proposition 1 still applies with $C \succ 0$. Similar reasoning holds for Proposition 2. Finally, the case of $C \prec 0$ or $Q \prec 0$, partially studied in [9], is not covered by this theory as in general one does not expect uniqueness of the NE. Consider, for example the game with costs (3), $N=2, m=1, C=1, Q=-1, c=0$ and $K_{1}=K_{2}=\{y \in \mathbb{R} \mid 0 \leq y \leq 1\}$. All the points $(0, s)$ and $(s, 0)$ with $0 \leq s \leq 1$ are Nash equilibria for this game.

\section{ALGORITHMS}

In the previous section we saw how, under suitable assumptions, finding a $\mathrm{NE}$ of $\mathcal{G}_{A}$ is equivalent to solving of a VI. In this section, we present projection based algorithms to solve such a variational inequality problem. We derive conditions for their convergence and show how they can be implemented in a decentralized fashion. Let us consider the $V I(K, T)$ with $T(\cdot)$ strictly monotone. Projection algorithms are known to converge in this case [10], but are slow or unusable when $N$ becomes large, as discussed in the following. Using the specific structure of game $\mathcal{G}_{A}$, we remedy the latter issue and prove convergence in subsection III-A by appropriately choosing the step size of the algorithm.

Definition 4 (Projection Operator): The operator $\operatorname{Proj}_{K}$ : $\mathcal{H} \rightarrow \mathcal{H}$, for $K \subset \mathcal{H}$ is defined as $\operatorname{Proj}_{K}(x):=$ $\arg \min _{z \in K}\|x-z\|_{2}$.

The following theorem guarantees convergence of the iteration defined in (6) to the solution of $V I(K, T)$, when $T$ is strictly monotone and Lipschitz [10].

Theorem 3: Consider the $V I(K, T)$ with $T: K \rightarrow \mathbb{R}^{n}$, where $K$ is a closed convex subset of $\mathbb{R}^{n}$. Furthermore assume that $T$ is strictly monotone and Lipschitz. Then the iteration

$$
\begin{gathered}
x^{k+1}=\operatorname{Proj}_{K}\left[x^{k}-\gamma_{k} T\left(x^{k}\right)\right] \\
\text { with } \quad \gamma_{k} \geq 0, \quad \lim _{k \rightarrow \infty} \gamma_{k}=0, \quad \sum_{k=0}^{\infty} \gamma_{k}=\infty .
\end{gathered}
$$

converges to the unique solution of $V I(K, T)$.

We refer to this as to the variable step Projection Algorithm. As we have seen previously, for the game $\mathcal{G}_{A}$ under the assumptions of Proposition 1, monotonicity and Lipschitz continuity are guaranteed for $T(x)$ in (4). Thus, (6) converges to the solution of the $\operatorname{VI}(K, T)$, i.e. the unique $\mathrm{NE}$ of $\mathcal{G}_{A}$.

Condition (7) requires $\gamma_{k} \rightarrow 0$ as $k \rightarrow \infty$ and may prevent to take a sufficiently large step size $\gamma_{k}$ when the iteration number $k$ increases. Thus, (6) may be slow to converge. For an example of this, see subsection IV-C.

\section{A. Fixed step Projection Algorithm}

In an attempt to avoid the previous problem, we consider the fixed step Projection Algorithm i.e. algorithm (6) with constant step size $\gamma$. The choice of $\gamma$ is crucial for the convergence. For a strictly monotone and Lipschitz operator $T(x)$, (6) converges when $\gamma$ is chosen to be sufficiently small, $0<\gamma<\bar{\gamma}$, see [10, Theorem 12.1.2]. A direct application of this theorem results in $\bar{\gamma} \sim \mathcal{O}(1 / N)$, and thus is of little use when $N$ is large, for example in the case of electricity markets, with a large number of participants. In the following, using the specific structure of the quadratic game of subsection II-C, we show convergence for the fixed step Projection Algorithm with improved upper bound $\bar{\gamma} \sim \mathcal{O}(1)$ when $N \rightarrow \infty$. In the derivation below we prove convergence for the affine cost (3). For simplicity assume 
that $Q$ and $C$ are symmetric. Let us introduce the definition of a contractive operator and a useful lemma.

Definition 5 (Contractive Operator): The operator $T(\cdot)$ : $\mathcal{H} \rightarrow \mathcal{H}$ is said to be contractive (resp. non expansive) if $\|T(x)-T(y)\|_{2} \leq L\|x-y\|_{2}$ with $L<1$ (resp. $\left.L \leq 1\right)$ $\forall x, y \in \mathcal{H}$.

Lemma 1: Given $C \in \mathbb{R}^{m \times m}$ symmetric, $i=1, \ldots, m$, $\operatorname{Spec}\left[\left(I_{N}+\mathbb{1}_{N} \mathbb{1}_{N}^{\top}\right) \otimes C\right]=\{(N+1) \lambda_{i}(C), \underbrace{\lambda_{i}(C), \ldots, \lambda_{i}(C)}_{N-1 \text { copies }}\}$,

Proof: Using the properties of the eigenvalues for Kronecker products, we know that $\operatorname{Spec}\left[\left(I_{N}+\mathbb{1}_{N} \mathbb{1}_{N}^{\top}\right) \otimes C\right]=$ $\left\{\mu_{j} \lambda_{i}(C)\right\} i=1, \ldots, m, j=1, \ldots, N$, where $\mu_{j}$ are the eigenvalues of $I_{N}+\mathbb{1}_{N} \mathbb{1}_{N}^{\top}$ counted with multiplicity. It is easy to see that

$$
\operatorname{Spec}\left[I_{N}+\mathbb{1}_{N} \mathbb{1}_{N}^{\top}\right]=\{N+1, \underbrace{1, \ldots, 1}_{N-1 \text { copies }}\} .
$$

Combining the two, one gets the desired result.

Proposition 3 (Improved convergence): Under the same hypothesis of Proposition 1, further assume $Q, C \in \mathbb{R}^{m \times m}$ symmetric. The fixed step Projection Algorithm

$$
x^{k+1}=\operatorname{Proj}_{K}\left[x^{k}-\gamma T\left(x^{k}\right)\right]
$$

converges to the unique solution of $V I(K, T)$ provided that $0<\gamma<\left(\lambda_{\max }(Q)+\frac{N+1}{N} \lambda_{\max }(C)\right)^{-1}$.

Proof: Finding a solution to the variational inequality $V I(K, T)$ is equivalent to finding a fixed point of the following map $H(x)=\operatorname{Proj}_{K}[x-\gamma T(x)]: K \rightarrow K \subset \mathbb{R}^{N m}$ [10]. We intend to show that $H(\cdot)$ is a contraction with respect to the euclidean $\|\cdot\|_{2}$ norm. The map $H(\cdot)$ is a composition of two operators: the $\operatorname{Proj}_{K}(\cdot)$ operator and the operator $G(\cdot) \doteq \mathrm{Id}-\gamma T(\cdot)$. Since projection is non expansive with respect to the 2-norm [14], it suffices to find $\gamma$ that renders $G(\cdot)$ contractive in the same norm.

In this case, the operator $T(x)=\left(B_{1}+B_{2}\right) x+b$ is linear with $B_{1}=2 I_{N} \otimes Q, B_{2}=\frac{2}{N}\left(I_{N}+\mathbb{1}_{N} \mathbb{1}_{N}^{\top}\right) \otimes C, b=\mathbb{1}_{N} \otimes 2 c$, and thus also $G(x)=B x-\gamma b, \quad B=\left(I_{m N}-\gamma\left(B_{1}+B_{2}\right)\right)$ is linear. As a consequence, the contractivity of $G(\cdot)$ is guaranteed when $L_{G}=\|\mathrm{D} G\|_{2}=\|B\|_{2}<1$. The matrix norm $\|B\|_{2}$ is given by the square root of the maximum singular value of $B$, which reduces to the spectral radius $\rho(G)=\max _{i}\left|\lambda_{i}(G)\right|$, thanks to symmetry of $Q, C$. We intend to find the values of $\gamma$ that make $\max _{i}\left|\lambda_{i}(G)\right|<1$, which is guaranteed if $\left|\lambda_{i}(G)\right|<1, \forall i$ or equivalently iff

$$
-1<1-\gamma \lambda_{i}\left(B_{1}+B_{2}\right)<1 \quad \forall i .
$$

Given that $B_{1}+B_{2} \succ 0$, the right hand inequality is satisfied for any $\gamma>0$. The inequality on the left hand side requires $\gamma \lambda_{i}\left(B_{1}+B_{2}\right)<2$, which is guaranteed if

$$
\gamma\left(\lambda_{\max }\left(B_{1}\right)+\lambda_{\max }\left(B_{2}\right)\right)<2 .
$$

Indeed $\lambda_{i}\left(B_{1}+B_{2}\right) \leq \lambda_{\max }\left(B_{1}+B_{2}\right) \leq\left(\lambda_{\max }\left(B_{1}\right)+\right.$ $\left.\lambda_{\max }\left(B_{2}\right)\right)$, where the last inequality is known as the Weyl's inequality [16], and thus $(10) \Longrightarrow \gamma \lambda_{i}\left(B_{1}+B_{2}\right)<2$ which implies the left hand side of (9) is satisfied. It follows that

$$
0<\gamma<2\left(\lambda_{\max }\left(B_{1}\right)+\lambda_{\max }\left(B_{2}\right)\right)^{-1} \Longrightarrow L_{G}<1,
$$

and the operator $H(x)$ is a contraction. As a consequence the Picard-Banach iteration (8) converges to the unique fixed point $x^{\star}$ of $H(x)$ [14], which is the solution to $V I(K, T)$ as previously discussed. It is trivial to see that $\lambda_{\max }\left(B_{1}\right)=2 \lambda_{\max }(Q)$, while using Lemma 1 one finds $\lambda_{\max }\left(B_{2}\right)=2 \frac{N+1}{N} \lambda_{\max }(C)$. Condition (11) reduces to $0<\gamma<\gamma_{M}=\left(\lambda_{\max }(Q)+\frac{N+1}{N} \lambda_{\max }(C)\right)^{-1}$ and thus concludes the proof.

With the same tools, one can prove a similar convergence result in the nonlinear case (1).

Remark 2: The value of $\gamma_{M}$ found here does not go to zero as $N \rightarrow \infty$. Thus, the result provides a convergence argument for any finite and arbitrary large $N$.

Remark 3: When $Q, C$ are symmetric $\mathcal{G}_{A}$ is a potential game, i.e. a minimizer of $P(x)=\frac{1}{2} x^{\top}\left(A_{1}+A_{2}\right) x+b^{\top} x$ is a NE of $\mathcal{G}_{A}$ [11]. Only in this case, distributed optimization algorithms can also be implemented, see for example [17].

\section{B. Decentralized implementation of projection algorithms}

When the number of players, $N$, is large, it becomes crucial to devise a decentralized implementation of projection algorithms. Let us consider the variable step algorithm and the cost function (3), as similar reasonings hold in the other cases. Given the explicit formula for $T(x)$ in (4), and that $K$ is a product space, equation (6) can be rewritten as

$x_{i}^{k+1}=\operatorname{Proj}_{K_{i}}\left[x_{i}^{k}-\gamma_{k}\left(\left(Q+Q^{\top}+\frac{2 C^{\top}}{N}\right) x_{i}^{k}+2 C \sum_{j=1}^{N} \frac{x_{j}^{k}}{N}+c\right)\right]$,

where $x_{i}^{k}$ represents the $k$-th iteration of the decision variable of the $i$-th player. The algorithm is implemented with the help of a central aggregator as follows. At the initial step, each player initializes $x_{i}^{0}$, sends it to an aggregator which computes $z^{0}=\sum_{i=1}^{N} x_{i}^{0} / N$ and broadcasts it to the players. Agents update $x_{i}^{k}$ according to (12) and the procedure iterates until convergence. The scheme is presented in the following table. Every player needs to know $N$, while $\gamma_{k}$ has to be the same across the population. This can be achieved, for instance, communicating the information at the initial step.

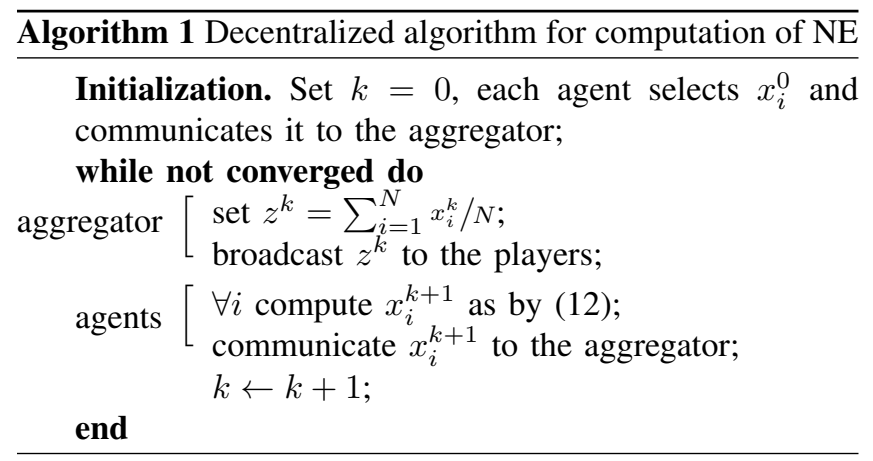

\section{CHARGING A FLEET OF PEVS}

\section{A. Formulation of the problem}

Let us consider the problem of managing the charge of a fleet of $N$ plug-in electric vehicles [4]. Each vehicle is equipped with a battery whose dynamics is taken to be

$$
s_{i}^{t+1}=s_{i}^{t}+a_{i} u_{i}^{t}, \quad i=1, \ldots, N, \quad t=0, \ldots, \tau-1,
$$


where $s_{i}^{t} \in[0,1]$ represents the state of charge (SOC) at time $t$. The constant $a_{i}$ describes the ratio between charging efficiency and battery size for the $i$-th PEV; $\tau \in \mathbb{N}$ indicates the length of the horizon. The initial SOC $s_{i}^{0}$ is known; while the final state of charge $s_{i}^{\tau}$ is dictated ahead of time by the particular needs of the owner of each PEV. Consequently, at every time step, each vehicle can choose how much charge to store in its battery by selecting $u_{t}^{i} \geq 0$, with the constraint of matching $s_{i}^{\tau}$. More realistic constraints could be implemented [8], but for simplicity, we use the formulation of [4]. The decision variable $u_{i}=\left[u_{i}^{0}, \ldots, u_{i}^{\tau-1}\right]$ is then constrained by $u_{i} \in \chi_{i}$, where

$$
\chi_{i}=\left\{u_{i} \geq 0 \mid a_{i} \sum_{t=0}^{\tau-1} u_{i}^{t}=s_{i}^{\tau}-s_{i}^{0}\right\} .
$$

We assume a time varying marginal price for the electricity, $p=\left[p_{0}, \ldots, p_{\tau-1}\right]$, where $p_{t}$ depends on the ratio between production and overall demand of electricity, at the same time $t$. This captures the fact that in future liberalized markets, the consumers can be given a price that depends on the demand of the power at that time. The total demand is considered to be the sum of the inflexible plus the aggregated PEVs' demand. The marginal price can be written as

$$
p_{t}=\mathrm{p}\left(\frac{1}{\kappa} \sum_{j=1}^{N} \frac{u_{j}^{t}}{N}+\frac{d_{t}}{\kappa}\right)
$$

where $\mathbb{p}: \mathbb{R} \rightarrow \mathbb{R}$ and $d_{t}, \kappa>0$ represent respectively the inflexible demand at time $t$, assumed to be known, and the total production capacity, both divided by $N$, see [4].

Each PEV is assumed rational and thus willing to find the sequence $u_{i}$ that minimizes its total electricity cost, while ensuring $u_{i} \in \chi_{i}$. Given the coupling of the players through the marginal cost, this results in the following game

$$
\min _{u_{i}} \sum_{t=0}^{\tau-1} p_{t} u_{i}^{t}, \quad \text { s.t. } u_{i} \in \chi_{i}, \quad i=1, \ldots, N .
$$

Our objective is to find a NE for the given game. The interest in Nash equilibria is twofold: first, it describes the natural situation in which rational players minimize their electricity bill; second, it has been shown in [4], that for $N \rightarrow \infty$ and homogenous population, the NE also minimizes the total cost of delivering the system demand and consequently posses the desired valley filling property. This means that the PEVs would maximize charging during the intervals in which the inelastic demand and therefore the price are lower.

\section{B. Linear and nonlinear marginal price $\mathrm{p}$}

We consider two price models: $\mathfrak{p}(r)=\beta r^{\delta}[\$ / \mathrm{kWh}], \beta=$ $0.15, \delta=1.5$, proposed in [4] and its linear approximation $\mathrm{p}(r)=\alpha r[\$ / \mathrm{kWh}], \alpha>0$. Let us first focus on the latter case, and note that the game takes the form

$$
\min _{u_{i}} 2\left(C \frac{1}{N} \sum_{j=1}^{N} u_{j}+c\right)^{\top} u_{i}, \quad \text { s.t. } u_{i} \in \chi_{i},
$$

where $C=\frac{\alpha}{2 \kappa} I_{N T} \in \mathbb{R}^{N \tau \times N \tau}$ and $c=\frac{\alpha}{2 \kappa}\left[d_{0}, \ldots, d_{\tau-1}\right]$. Recall that the constraint set $\chi_{i}$ of (13) is a closed and convex subset of $\mathbb{R}^{\tau}$. Consequently this problem falls into the more general aggregative game $\mathcal{G}_{A}$ introduced in section II-A, by selecting $Q=0$ in equation (3). As a consequence, since $C=\frac{\alpha}{2 \kappa} I_{N \tau} \succ 0$, Proposition 1 guarantees the existence and uniqueness of a Nash equilibrium for any finite $N$. Furthermore, the variable step Projection Algorithm of equation (6) converges as Theorem 3 certifies. The convergence is guaranteed also for the fixed step Projection Algorithm of equation (8), by choosing $0<\gamma<2 \frac{N}{N+1} \frac{\kappa}{\alpha}$, see Proposition 3. This is in contrast to [4], [9] where an artificial quadratic term is introduced to show convergence to an $\varepsilon$-Nash equilibrium. In the case of $\mathrm{p}(r)=0.15 r^{1.5}$, the game reduces to (1) with $g_{l}(z)=0.15\left(\frac{1}{\kappa} \sum_{j=1}^{N} \frac{u_{j}^{l}}{N}+\frac{d_{l}}{\kappa}\right)^{1.5}$ for $l=1, \ldots, \tau$ and $Q=0$. The conditions of Proposition 2 are satisfied and with $N$ sufficiently large, as for the following simulations, the associated VI is strictly monotone, thus ensuring existence and uniqueness of the NE.

\section{Simulation results}

In this section we present various numerical results, with the objective of comparing algorithms (6) and (8), for different populations of electric vehicles. We first consider the case of a homogeneous population i.e. a population of identical PEVs both in terms of battery dynamics and in terms of SOC requirements. The parameters of the simulations are taken from [4] and listed in the following table for reference. The inflexible demand $d_{t}$ (see Figure 2) shows the typical load of a summer day in 2007 of the Midwest USA ISO region. The number of vehicles is chosen as $N=10^{7}$. This corresponds to a penetration of approximately $30 \%$ of PEVs in the area. The charging period is taken to be 24 hours, starting from 12:00 pm. The linear marginal price function $\mathrm{P}_{L}(r)=0.12 r$ is considered as a good approximation of $\mathrm{P}_{N}(r)=0.15 r^{1.5}$ and used in the following simulations. The resulting upper bound on the step becomes $\gamma_{M}=20 \frac{N}{N+1}$. The initial condition $x_{i}^{0}$ is randomly chosen for every vehicle.

\begin{tabular}{|c|c|c|c|c|c|}
\hline Parameter & $N$ & $\kappa$ & $s_{i}^{0}$ & $s_{i}^{T}$ & $a_{i}$ \\
\hline Value & $10^{7}$ & $12 \mathrm{~kW}$ & 0.15 & 1 & $0.085(\mathrm{kWh})^{-1}$ \\
\hline
\end{tabular}

Figure 1 presents the convergence profile of $z=$ $\frac{1}{N} \sum_{j=1}^{N} u_{j}$ for fixed and variable step Projection Algorithm with $\gamma=17$ and $\gamma_{k}=17 k^{-0.5}$. The NE is identified by $z^{\star}$.

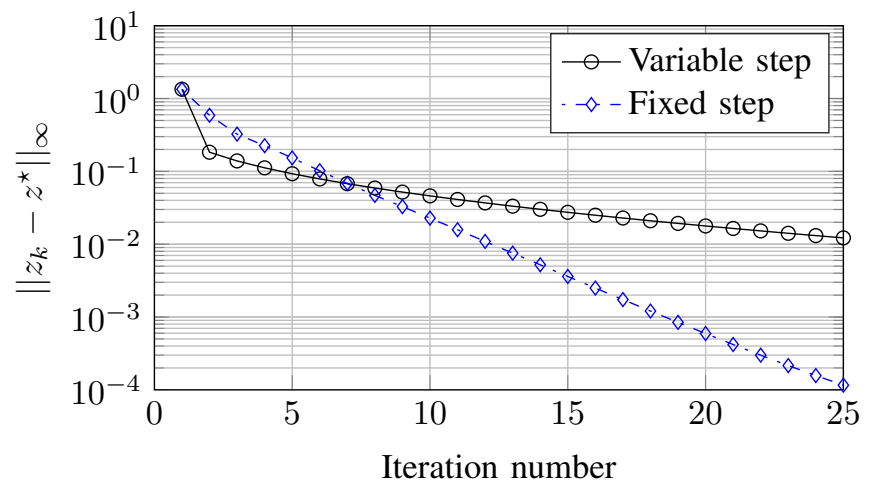

Fig. 1: Convergence profile for (8) and (6). 
The convergence for (8) is monotonic and exponential, in agreement with Proposition 3, while the convergence profile of (6) depends considerably on the choice of the sequence $\left\{\gamma_{k}\right\}_{k=0}^{\infty}$. Since by condition (7) when $k \rightarrow \infty$ the step $\gamma_{k}$ converges to zero, this may result in a slower algorithm as the number of iteration increases. We have observed faster convergence when $\gamma_{k}$ was taken of the form $\gamma_{k} \sim \mathcal{O}\left(k^{-\beta}\right)$ for small values of $\beta$, but this results in a step that is almost constant between iterations thus reducing (6) to (8). Convergence to the Nash equilibrium is guaranteed using much fewer iterations if compared to the previous work of [4] and [9], where they prove convergence to an $\varepsilon$-Nash equilibrium, with $\varepsilon \sim \mathcal{O}(1 / N)$. At each iteration the players communicate $x_{i}^{k}$ to the aggregator and the aggregator returns $\frac{1}{N} \sum_{j=1}^{N} x_{j}^{k}$. Faster convergence results in less communication.

Figure 2 details the convergence history of (8) with $\gamma=17$ for the homogenous scenario just introduced. The dashed curve represents the initial condition, while successive iterations are presented in blue. The last iteration corresponding to the NE is drawn with round markers and has the property of being valley filling, in accordance with [4].

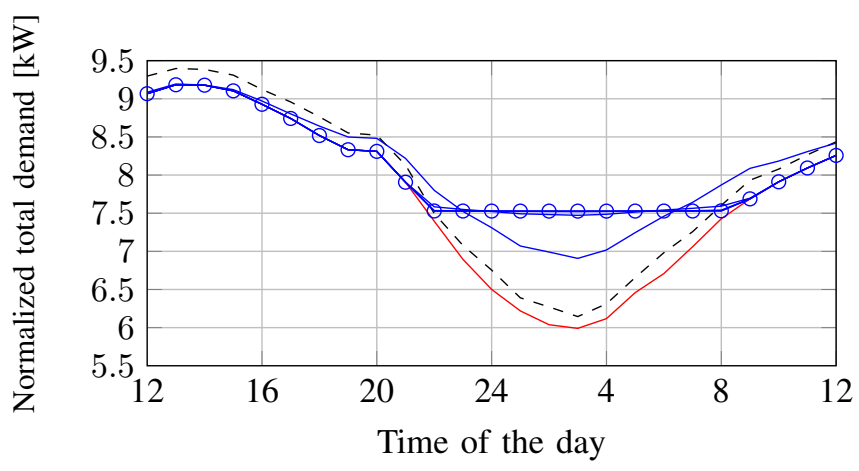

Fig. 2: Convergence history for (8). The inflexible demand $d_{t}$ is shown in red.

Figure 3 presents the comparison of the convergence speed for the homogeneous population just discussed and the case of heterogeneous players for both linear and non linear marginal prices. For the latter $a_{i} \sim \mathrm{U}([0.08,0.09])$ and $s_{i}^{T}-s_{i}^{0} \sim \mathrm{U}([0.3,0.8])$, while the rest of the parameters are left unchanged. We have selected $\gamma=10$ to compare these scenarios, but a different step size for each case could perform better. In general, the convergence appears faster for the non linear price while heterogeneity slows down the algorithm. In all the cases the convergence is exponential.

\section{CONCLUSIONS}

We studied the problem of existence and uniqueness of Nash equilibria for a certain class of aggregative games. Leveraging on the specific structure of the player's costs and constraints, we derived sufficient conditions to guarantee that a unique NE exists. The derivation was based on connecting the set of Nash equilibria with the solutions of a certain variational inequality. For finite and arbitrary large population we presented two algorithms that converge to the NE in a decentralized fashion. We concluded with an application to charging of PEVs in a liberalized electricity market, and

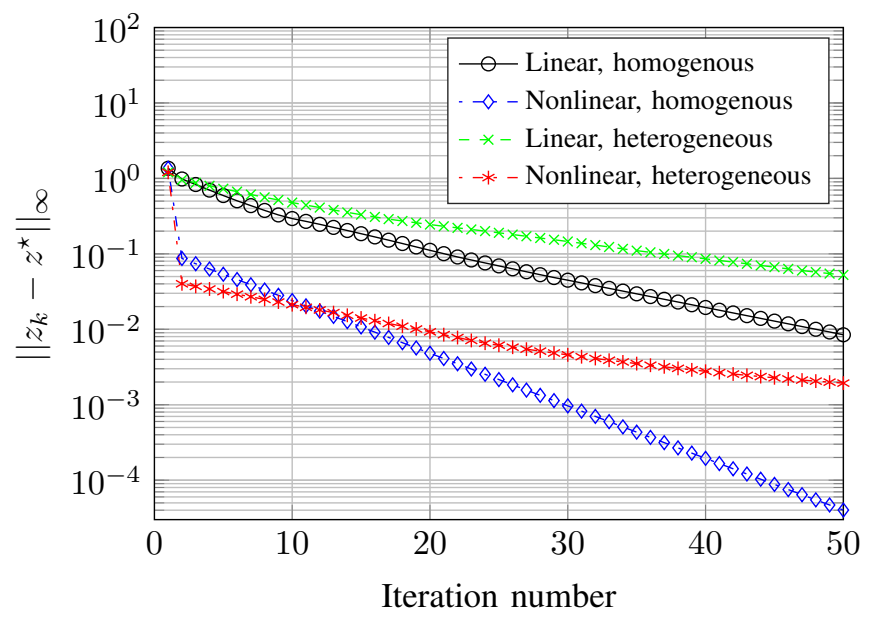

Fig. 3: Convergence profile for iteration (8), $\gamma=10$.

showed improved convergence speed. We are extending the algorithms to address asynchronous implementations and to include coupling constraints. These extensions will make the model more suitable for real world applications.

\section{REFERENCES}

[1] D. D. Siljak, Decentralized control of complex systems. Courier Corporation, 2011.

[2] J.-M. Lasry and P.-L. Lions, "Mean field games," Japanese Journal of Mathematics, vol. 2, no. 1, pp. 229-260, 2007.

[3] M. Huang and P. E. Caines, "The NCE (Mean Field) Principle With Locality Dependent Cost Interactions," IEEE Transactions on Automatic Control, vol. 55, no. 12, pp. 2799-2805, 2010.

[4] Z. Ma, D. S. Callaway, and I. Hiskens, "Decentralized charging control of large populations of plug-in electric vehicles," Control Systems Technology, IEEE Transactions on, vol. 21, no. 1, pp. 67-78, 2013.

[5] R. Couillet, S. M. Perlaza, H. Tembine, and M. Debbah, "A mean field game analysis of electric vehicles in the smart grid," 2012 Proceedings IEEE INFOCOM Workshops, pp. 79-84, 2012.

[6] H. Chen, Y. Li, R. H. Louie, and B. Vucetic, "Autonomous demand side management based on energy consumption scheduling and instantaneous load billing: An aggregative game approach," Smart Grid, IEEE Transactions on, vol. 5, no. 4, pp. 1744-1754, 2014.

[7] Z. Ma, I. Hiskens, and D. Callaway, "A decentralized MPC strategy for charging large populations of plug-in electric vehicles," IFAC Proceedings Volumes (IFAC-PapersOnline), vol. 18, 2011.

[8] L. Gan, U. Topcu, and S. Low, "Optimal Decentralized Protocol for Electric Vehicle Charging," IEEE Transactions on Power Systems, vol. 28, no. 2, pp. 940-951, 2013.

[9] S. Grammatico, F. Parise, M. Colombino, and J. Lygeros, "Decentralized convergence to nash equilibria in constrained mean field control," IEEE Trans. on Automatic Control (accepted), 2014.

[10] F. Facchinei and J.-S. Pang, Finite-dimensional variational inequalities and complementarity problems. Springer Science \& Business Media, 2007.

[11] M. K. Jensen, "Aggregative games and best-reply potentials," Economic theory, vol. 43, no. 1, pp. 45-66, 2010.

[12] T. Basar and G. J. Olsder, Dynamic noncooperative game theory. SIAM, 1995, vol. 200.

[13] G. Stampacchia, "Variational inequalities," Theory and applications of monotone operators, pp. 101-192, 1969.

[14] H. H. Bauschke and P. L. Combettes, Convex analysis and monotone operator theory in Hilbert spaces. Springer Science \& Business Media, 2011.

[15] G. Scutari, D. P. Palomar, F. Facchinei, and J.-S. Pang, "Monotone games for cognitive radio systems," in Distributed Decision Making and Control. Springer, 2012, pp. 83-112.

[16] H. Weyl, "Das asymptotische verteilungsgesetz der eigenwerte linearer partieller differentialgleichungen," Mathematische Annalen, vol. 71, no. 4, pp. 441-479, 1912.

[17] D. P. Bertsekas, Nonlinear Programming. Athena Scientific, 2004. 